\title{
EVALUACIÓN DEL RENDIMIENTO POTENCIAL DE CUATRO CULTIVARES DE BATATA (Ipomoea batatas (L.) LAM.) EN CORRIENTES
}

\section{Assessment of potential yield of four sweet potatoes cultivars in Corrientes}

\author{
González, César A.; Giménez, Laura I.; Burgos, Angela M. \\ Universidad Nacional del Nordeste. Facultad de Ciencias Agrarias. \\ cesaragrogonzalez@gmail.com; laugim@yahoo.com; burgosangela@hormail.com
}

\section{RESUMEN}

La batata [Ipomoea batatas (L.) Lam., familia Convolvulaceae] se cultiva en los cinco continentes, ubicándose en el séptimo lugar entre los cultivos alimenticios y en el tercero de los que tiene órganos comerciales subterráneos. En Argentina, el rendimiento promedio es de 14,9 tn ha ${ }^{-1}$, lo que se considera bajo respecto a otros países de Latinoamérica; particularmente en la provincia de Corrientes la batata se produce con tecnología obsoleta. El objetivo del trabajo ha sido conocer el potencial de rendimiento de raíces de batata de cuatro cultivares bajo manejo tecnificado, en las condiciones agroecológicas del norte de la Provincia de Corrientes. A este fin el experimento se llevó a cabo aplicando fertilización, control integrado de malezas y riego localizado. Los cultivares (cvs) saneados y de interés comercial sometidos a evaluación han sido: Beauregard y Arapey INIA, de ciclo corto y Morada INTA y Okinawa 100 de ciclo largo. Para ello se utilizó un diseño en bloques completos al azar, con cuatro repeticiones, registrándose variables como Número de Raíces Totales (NRT), Comerciales (NRC) y de No Comerciales (NRNC), Peso Fresco Total de Raíces (PFT), de las Comerciales (PFRC) y las No Comerciales (PFRNC) por $\mathrm{m}^{2}$ a partir de los cual se realizaron estimaciones por hectárea. Los resultados fueron sometidos al análisis de la varianza (ANOVA) y prueba de Tukey $(\mathrm{p} \leq 0,05)$. Los $c v s$ Okinawa 100 y Morada INTA, alcanzaron los mayores rendimientos con 72,79 y 55,29 tn ha ${ }^{-1}$ respectivamente, quintuplicando y triplicando el rendimiento promedio de la Provincia de Corrientes. Los cvs Beauregard y Arapey INIA rindieron 36,42 y 33,03 tn ha ${ }^{-1}$ respectivamente, duplicando el rendimiento promedio provincial. El NRC y el NRNC no difirieron estadísticamente entre los cultivares evaluados.

Palabras clave: Boniato - Camote - Cosecha - Raíces tuberosas.

\begin{abstract}
Sweet potatoe [Ipomoea batatas (L.) Lam., Convolvulaceae family] is cultivated in the five continents, it is the seventh most cultivated food crop in the world and the third with commercial underground organs. In Argentina, the average yield is 14,9 tn ha-1, which is considered low compared to other Latin American countries; particularly in the province of Corrientes sweet potatoe is produced with obsolete technology. The objective of this work has been to know the roots potential yield of four sweet potatoes cultivars under technical management in the agro-ecological conditions of the north of Corrientes Province. For this purpose, the experiment was carried out applying fertilization, integrated weed management and localized irrigation. The evaluated sanitized cultivares (cvs) of commercial interest had been: for short cycle Beauregarr and Arapey INIA, and for long cycle Morada INTA and Okinawa 100. For this, a randomized complete block design was used with four repetitions measuring variables such as: Number of Total (NRT), Commercial (NRC) and Non-Commercial (NRNC) Roots, Total Fresh Weight of Roots (PFT) of Commercial Roots (PFRC) and Non-Commercial Roots (PRNC) per $\mathrm{m}^{2}$ from which estimates were made per hectare. The results were submitted to the analysis of variance (ANOVA) and Tukey's test $(\mathrm{p}<0.05)$. The $c v s$ Okinawa 100 and Morada INTA achieved the highest yields with 72,79 and 55,29 tn ha- respectively, five-fold and three-fold the average yield of the Province of Corrientes was increased. The $c v s$ Beauregard and Arapey INIA achieved 36.42 and 33,03 tn harespectively, two-fold increased the provincial average yield. The NRC and the NRNC did not differ statistically between the cultivars evaluated.
\end{abstract}

Keywords: Sweet potato- Harvest -Tuber Roots.

Recibido: 11/jun/2020. Aceptado: 27/jul/2020 


\section{INTRODUCCIÓN}

La batata [Ipomoea batatas (L.) Lam.] es una especie herbácea y perenne, sin embargo, es cultivada como una planta anual utilizando raíces reservantes y esquejes (guías) para su propagación vegetativa. La sistemática la incluye dentro de la familia de las Convolvuláceas dentro de las cuales también se encuentran trece especies silvestres emparentadas. Su hábito de crecimiento es predominantemente postrado, pero puede variar entre erecto, semi-erecto, extendido y muy extendido. La especie incluye plantas muy variables en su morfología. Los tallos son cilíndricos verdes a totalmente pigmentados con antocianinas (rojo-morado). La longitud de los mismos puede variar de acuerdo al hábito de crecimiento que van desde un metro en las erectas hasta los cinco metros en las muy extendidas (Huamán, 1992).

Las hojas son simples y presentan diversidad de formas que pueden ser redondeadas, cordadas, reniformes, lobuladas y casi divididas. El color es generalmente verde, pero puede presentar pigmentación morada sobre todo en las más jóvenes (Martí et al., 2014).

Su sistema radicular consiste en raíces fibrosas encargadas de la absorción de nutrientes y agua. Raíces tipo lápiz, con leve engrosamiento y lignificación y raíces reservantes en las que se almacenan fotoasimilados. Éstas últimas se originan debido a la presencia de un cambium anómalo que genera un tejido parenquimático rico en almidón, (Martí et al., 2014). Las formas y tamaños de las raíces reservantes pueden variar según el cultivar desde redonda a elíptica, ovada, oblonga e irregular. El color de la piel de las raíces puede ser blanco, crema, amarillo, anaranjado, morado y rosado. El color de la pulpa puede ser blanca, crema, amarilla, anaranjada y en algunos casos con incrustaciones moradas (Huamán, 1992; Martí, et al., 2014). Desde el punto de vista comercial se clasifican por su tamaño en grandes (mayor a 500 gramos), medianas (entre 250 y 500 gramos) y chicas (entre 60 y 250 gramos), (SENASA, 1983).

El ciclo total es variable según cultivares y se desarrolla entre 90 y 150 días (Martí et al., 2014).

A nivel global se cultiva en los cinco continentes. Dentro de los cultivos destinados a la alimentación se ubica en el séptimo lugar y en el tercero de los que tiene órganos comerciales subterráneos luego de la papa y la mandioca (Loebenstein et al., 2009). La producción mundial ronda los 92 millones de toneladas con una superficie de 8 millones de hectáreas cosechadas, dando un rendimiento promedio a nivel mundial de 11,4 tn ha ${ }^{-1}$. Aproximadamente el 95\% de esa superficie se sitúa en países de Asia (49\%) y África (46\%) en los que es considerado un cultivo de subsistencia. La producción mundial se concentra en Asia con un 75\% del total, del cual el 41\% corresponde a China (FAOSTAT, 2019). En América del Sur se relevaron 113.390 ha con un rendimiento promedio de 13,57 tn ha ${ }^{-1}$, levemente superior al mundial.

En Argentina, la producción de batata es de 339.359 tn distribuidas en 22.772 ha (FAOSTAT, 2019) con lo que se alcanza un rendimiento promedio de $14,9 \mathrm{tn} \mathrm{ha}^{-1}$. Si se tiene en cuenta que, en países más lejanos, pero en una latitud semejante como es Australia, el rendimiento promedio llega a 36,4 tn ha ${ }^{-1}$ (FAOSTAT, 2019), no caben dudas que hay factores de la producción que deben ser estudiados para poder superar el promedio nacional y aproximarnos a los potenciales que el cultivo ofrece.

La plasticidad de la especie permite que pueda ser cultivada a lo largo y lo ancho de nuestra geografía nacional. La región pampeana (Buenos Aires, Córdoba y Santa Fe) y la del noreste (Corrientes, Formosa, Chaco, Misiones y Entre Ríos) representan el $83 \%$ de la superficie cultivada (43\% para la primera zona y $40 \%$ para la segunda) del país. El noroeste (Santiago del Estero, Tucumán, Salta, Catamarca y Jujuy) posee el $15 \%$ y el restante 2\% corresponde a Cuyo (Mendoza, San Juan y San Luis) (Martí, 2008).

Particularmente en la provincia de Corrientes, la batata es un cultivo que está presente en la chacra de todos los pequeños productores. En algunas colonias constituye el principal cultivo de renta, mientras que en otras se la cultiva para consumo familiar o bien como forraje para distintas especies animales (Pletsch, 2006). En 2015, de 1.675 hectáreas se cosecharon 21.785 toneladas dando un rendimiento promedio de 13 tn ha ${ }^{-1}$ (Gauna, 2016). Los últimos datos provistos por Araujo et al. (2019) ponen de manifiesto una reducción abrupta a 350 ha con un leve incremento de rendimientos promedios que están a la par del rendimiento nacional $\left(14,8 \mathrm{tn} \mathrm{ha}^{-1}\right)$. Este cultivo se encuentra frecuentemente en sistemas productivos diversificados donde integra rotaciones que incluyen maíz, mandioca, porotos y se combinan con la producción cítrica y ganadera. 
En Corrientes, el $42 \%$ de los productores no fertiliza el cultivo, el $53 \%$ circunscribe el control de malezas al método manual a través de carpidas seguido por un $23 \%$ que combinan un esquema de herbicidas y control manual y tan solo el 9\% de los productores utiliza material de propagación saneado (Araujo et al., 2019).

En la agricultura, donde se usan grandes cantidades de agua, se producen también grandes pérdidas sobre todo en la utilización de métodos de riego superficial donde el escurrimiento y la percolación son generalmente significativos. Frente a este suceso surge la necesidad de implementar mejoras en el riego. Por ello, resulta imprescindible poner en práctica nuevas formas de aplicación del agua de riego que permitan optimizar el uso del agua y lograr obtener mayor productividad y mayor rendimiento de los cultivos (Delgado y Serna, 2018).

Algunos países como Honduras han implementado tecnologías como ser riego localizado (goteo), sistema que provee un mejor control de malezas, proporciona un microclima ideal en la zona radicular y mejora la calidad del producto en cuento a uniformidad en los tubérculos y buen color de la piel, con la finalidad de lograr los estándares de calidad para la exportación del mismo (León et al., 2013).

La batata produce rendimientos más que aceptables en suelos donde otros cultivos no prosperan a menos que se los fertilice. No obstante, se observan respuestas a la fertilización con niveles bajos de fósforo y potasio (Martí, 2018). Del Valle (2017), afirma que la fertilización ajustada mejora la producción de batata y los ingresos del productor.

En relación a los patógenos, las virosis son las patologías más importantes que afectan el cultivo de batata a nivel global, constituyendo potencialmente la limitante de la producción de mayor relevancia. La utilización de plantines saneados es el único modo eficiente, económico e inmediato de control de estas virosis de batata (Di Feo, 2015).

Teniendo en cuenta lo anteriormente expuesto se realizó una propuesta de manejo tecnificado atendiendo diversos factores que pueden modificarse para aumentar la productividad de un cultivo bajo un ambiente determinado. Dentro de estos, cinco han sido aplicados en este ensayo: $i$ ) la fertilización, fundamental para el crecimiento, desarrollo, y producción; ii) el control integrado de malezas a fin de posicionar al cultivo en la captación de los recursos del ambiente sin competencia o con competencia reducida a una mínima expresión; iii) el riego localizado; iv) el cultivar, los más utilizados a nivel comercial en la provincia de Corrientes son de piel blanca y pulpa blanco-crema como Okinawa 100. También se pueden encontrar frecuentemente cultivares de piel amarillo claro y pulpa amarillo crema o anaranjado como Sombrerito1 o Pionera. Con menor frecuencia se encuentran cultivares de piel morada y pulpa amarilla anaranjada como Arapey INIA Morada INTA o Mechada. Los cultivares de piel y pulpa anaranjada como Beauregard, Gem, Covington o Boni INTA solo se encuentran a nivel experimental, pese a que actualmente son considerados como alimentos biofortificados por poseer diez veces más carotenoides totales que los elaborados a partir de cultivares comunes (Arguedas-Gamboa et al., $2015)$; v) el uso de material saneado. Este último, pero no por ello menos importante, dado que la propagación vegetativa de la especie hace que la acumulación y perpetuación de virus sea potencialmente la principal limitante de la producción en cualquier zona productora (Martí et al., 2014). La utilización de esta tecnología permite una recuperación del tipo varietal, de los rendimientos propios de cada clon y de la calidad del producto (Di Feo, 2015).

El manejo de todos estos factores podría contribuir a alcanzar un rendimiento, que asegure la rentabilidad del cultivo.

El objetivo del presente trabajo fue conocer el potencial de rendimiento de raíces de batata de cuatro cultivares contrastantes cultivados bajo condiciones de manejo tecnificado en las condiciones agroecológicas del norte de la Provincia de Corrientes.

\section{MATERIALES Y MÉTODOS}

\section{Lugar:}

Los ensayos se realizaron en el predio del Centro Tecnológico de Producción (CE.TE.PRO) del Ministerio de Producción de Corrientes. Ruta Nacional N 12 Km 1032. Corrientes Capital, República Argentina. Coordenadas: ( $27^{\circ} 28^{\prime}$ Lat. S y $58^{\circ} 16^{\prime}$ Long. O). 


\section{Caracterización edáfica:}

El suelo es un Udipsament árgico, pertenece a la serie Ensenada Grande cuyas características son: susceptibilidad a la erosión eólica e hídrica y baja fertilidad. En superficie textura arenosa-franco y de reacción neutra. Presenta un horizonte enterrado, argílico, Btb, franco-arcillo-arenoso que continua a más de $115 \mathrm{~cm}$. Son profundos, de buenas condiciones físicas para el desarrollo radical y penetración del agua pero son suelos de baja fertilidad y de baja retención de humedad y la materia orgánica, es de menos del 1\% en capa arable con muy pobres niveles de bases de cambio, aunque aumentan en profundidad (Escobar et al., 1996).

\section{Caracterización climática:}

La provincia de Corrientes posee un clima subtropical, muy cálido en verano pero con heladas en invierno. Tiene características de clima húmedo, con frecuentes excesos hídricos en otoño y primavera, y moderados y eventuales déficits, principalmente en verano. Según el sistema de Köppen la clasificación para todas las localidades de la provincia es: Cf w’a (h) que expresa un clima mesotermal, cálido templado, sin estación seca con precipitación máxima en otoño, y verano muy cálidos con temperaturas superiores a $22{ }^{\circ} \mathrm{C}$ y media anual superior a $18{ }^{\circ} \mathrm{C}$. Se clasifica el régimen térmico como hipertérmico por poseer una temperatura media de suelo (a $50 \mathrm{~cm}$ ) anual superior a $22^{\circ} \mathrm{C}$ y una amplitud térmica anual mayor de $5^{\circ} \mathrm{C}$ (régimen no "iso").

El régimen hídrico es údico: en la mayoría de los años no se seca por más de 90 días consecutivos (Van Wambeke y Scoppa, 1976 citado por Escobar et al., 1996).

Finalmente, las condiciones climáticas citadas resultan propicias para el cultivo de batata en Corrientes.

\section{Manejo del experimento:}

Preparación del suelo, construcción de camellones y plantación:

El ensayo fue realizado durante la campaña 2019/20, aproximadamente 30 días antes de la plantación se realizó una pasada de rastra de discos. Pasados 20 días se realizó una segunda pasada de rastra. Por último, diez días antes de plantar se construyeron los camellones utilizando un arado de reja y vertedera. Éstos fueron de 0,3 $\mathrm{m}$ de altura por $0,5 \mathrm{~m}$ de ancho y $12 \mathrm{~m}$ de largo.

La plantación se realizó en el mes de octubre. Se utilizaron guías de 0,35 m de longitud. La densidad de plantación fue de 33.333 plantas ha ${ }^{-1}(0,3 \mathrm{~m} \mathrm{x} \mathrm{1,0} \mathrm{m).} \mathrm{El} \mathrm{hoyado} \mathrm{y} \mathrm{la} \mathrm{plantación} \mathrm{se} \mathrm{realizaron} \mathrm{de} \mathrm{forma} \mathrm{manual.}$

\section{Material biológico saneado:}

El material biológico utilizado para la evaluación provino de la colección de cultivares saneados del Vivero Hortícola del Centro Tecnológico de Producción CE.TE.PRO del Ministerio de Producción de Corrientes, cuyo origen (plantas madres) es el Instituto de Patología Vegetal del Centro de Investigaciones Agropecuarias del Instituto Nacional de Tecnología Agropecuaria (IPAVE-CIAP-INTA), Córdoba.

Para el presente trabajo se escogieron cuatro cultivares de importancia comercial y genética contrastante:

- $c v$ Arapey INIA (ARA). Es de origen uruguayo. Es uno de los más utilizados en Argentina. De piel morada y pulpa crema amarilla con vetas naranjas. Hojas cordadas verdes. Ciclo de 90 días.

$-c v$ Beauregard (BEA). Es de origen estadounidense y el más utilizado allí. De piel cobriza y pulpa anaranjada. Hojas cordiformes y terminadas en punta verdes de adultas y moradas cuando jóvenes. Ciclo de 90 días.

$-c v$ Morada INTA (MOR). Es de origen argentino. Fue el cultivar más utilizado en Argentina. De piel morada y pulpa amarilla con inclusiones naranja. Hojas penta-lobadas, verdes. Ciclo de 150 días.

- cv Okinawa 100 (OKI). Es de origen japonés. Ampliamente difundido en la provincia de Corrientes. De piel crema y pulpa blanca. Hojas cordadas verdes. Ciclo de 150 días.

\section{Fertilización:}

Si bien la batata es un cultivo muy rústico que se adapta a una amplia gama de situaciones ambientales y que produce rendimientos más que aceptables en suelos donde otros cultivos no prosperan a menos que se los fertilice (Ames et al., 1997. Martí et al., 2014), sin dudas para alcanzar rendimientos cercanos a los potenciales 
debe proporcionarse una nutrición balanceada en función de los requerimientos. Debido a que el suelo de la parcela presentaba limitaciones nutricionales (Tabla 1) y a la baja fertilidad asociada a la serie Ensenada Grande antes mencionada se aplicó un plan de fertilización que respondió a los requerimientos de la especie citados por Ames et al. (1997) para un rendimiento estimado de $30 \mathrm{tn} \mathrm{ha}^{-1}$.

Tabla 1. Análisis químico del suelo del sitio de experimentación. Metodología: N (Semi Micro Kjeldah) $\mathrm{P}$ (Bray I). Ca, Mg y K (Acetato de amonio pH 7). MO (Walkley y Black. pH: agua destilada suelo: 2.5-1).

\begin{tabular}{|c|c|c|c|c|c|c|c|c|}
\hline$\overline{\text { pH }}$ & $\overline{\mathbf{N}}$ & $\overline{\mathbf{P}}$ & $\bar{K}$ & $\overline{C a}$ & $\overline{M g}$ & $\mathbf{N a}$ & MO & $\mathbf{\Omega}$ \\
\hline & $\%$ & $\overline{p p m}$ & & meq/ & $00 \mathrm{~g}$ & & $\%$ & $\mathrm{dS} / \mathrm{m}$ \\
\hline 5,33 & 0,036 & 30,9 & 0,08 & 2,32 & 0,16 & 0,05 & 0,22 & 0,472 \\
\hline
\end{tabular}

\section{Riego:}

Si bien las precipitaciones registradas durante el ciclo del cultivo fueron suficientes en términos absolutos para el desarrollo del mismo (Figura 1), la distribución no fue uniforme y se registraron periodos de déficits hídricos. Esto ocurrió durante la $1^{\mathrm{a}}$ quincena de noviembre y $2^{\mathrm{a}}$ de diciembre, $1^{\mathrm{a}}$ de enero y todo el mes de marzo. Con el objeto de mantener al cultivo en condiciones óptimas de humedad de suelo durante todo su ciclo, se aplicó riego localizado de alta frecuencia (goteo) en cuatro oportunidades. Se utilizaron cintas con goteros a 10 $\mathrm{cm}$. Cada riego de 30 minutos de duración correspondió a un volumen de agua de $10 \mathrm{~L} \mathrm{~m}$.

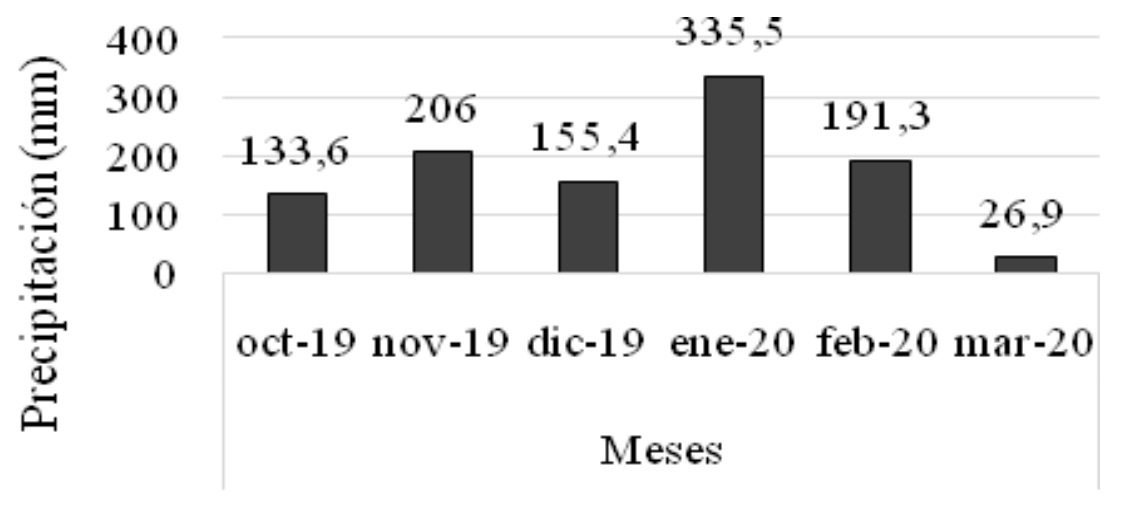

$\square$ Precipitaciones mensuales (mm)

Figura 1. Precipitaciones mensuales en milímetros ( $\mathrm{mm})$ durante el ciclo del cultivo. Fuente: Instituto Correntino del Agua y el Ambiente (ICAA).

\section{Manejo de Malezas:}

Con el objetivo de mantener libre de malezas la parcela, se optó por un manejo integrado que consistió en partir de la aplicación del herbicida preemergente Dual Gold ${ }^{\circledR}$, S-Metolacloro $(96 \%$ p/v) a dosis media $(900$ $\mathrm{cm}^{3} \mathrm{ha}^{-1}$ ) luego de la plantación. Posteriormente para el control de gramíneas, se realizó la aplicación del herbicida postemergente selectivo Sheriff ${ }^{\circledR}$, Quizalofop-p-etil $(1,8 \% \mathrm{p} / \mathrm{p})$ a dosis media de $\left(1000 \mathrm{~cm}^{3} \mathrm{ha}^{-1}\right)$. Todo se complementó con carpidas manuales durante el ciclo del cultivo.

\section{Diseño del Experimento:}

Se utilizó un diseño en bloques completos al azar, con cuatro repeticiones. Cada parcela experimental estuvo conformada por cuatro líneos, de los cuales solo los dos centrales fueron utilizados para realizar mediciones y muestreos. Cada parcela tuvo una superficie de $9 \mathrm{~m}^{2}$ ( $\left.3 \mathrm{~m} \mathrm{x} 3 \mathrm{~m}\right)$. Para las mediciones se tomaron las muestras de $1 \mathrm{~m}^{2}$ centrales de cada repetición. 


\section{Variables medidas:}

La cosecha de raíces se realizó en dos oportunidades: los cultivares Okinawa 100 y Morada INTA a los 150 DDP y los cultivares Arapey INIA y Beauregard a los 90 DDP. En esa instancia se cuantificaron los componentes numéricos del rendimiento de raíces por medio del conteo del Número de Raíces Totales (NRT) y separadas en dos categorías: Número de Raíces Comerciales (NRC) y Número de Raíces No Comerciales (NRNC). También se realizó la determinación del Peso Fresco Total (PFT) y de cada categoría Peso Fresco de Raíces Comerciales (PFRC) y Peso Fresco de Raíces No Comerciales (PFRNC), recolectadas de $1 \mathrm{~m}^{2}$ de cada una de las 4 parcelas experimentales. A partir de los datos registrados en el experimento en $\mathrm{g} \mathrm{m}^{2}$ de superficie, se realizaron las estimaciones de las variables por hectárea.

\section{Análisis Estadístico:}

Se realizó un análisis de la varianza (ANOVA) para todas las variables evaluadas, previa determinación de la normalidad y heterogeneidad de varianzas. Se realizó la prueba de Tukey para comparar las medias entre los tratamientos $(\mathrm{p} \leq 0,05)$ usando el software InfoStat versión 2019.

\section{RESULTADOS Y CONSIDERACIONES FINALES}

En virtud de los resultados encontrados (Tabla 2) se evidencia que el PFRC y el PFRNC en tn ha ${ }^{-1}$ no difirió estadísticamente entre los cultivares, pero la sumatoria de ambos que da el PFT (tn ha $^{-1}$ ) puso de manifiesto diferencias estadísticas. Estas diferencias alcanzaron a ser significativas entre el $c v$ Arapaey INIA y el $c v$ Okinawa 100, este último superó en más del 100\% al primero. Los cultivares Beauregard y Morada INTA que asumieron valores intermedios, no se diferenciaron entre ellos y respecto de Okinawa 100, la diferencia no llegó a ser significativa, pero en términos de valores absolutos el $c v$ Morada INTA alcanzó un 53\% más que el $c v$ Beauregard, y el $c v$ Okinawa 32\% más que el $c v$ Morada INTA.

Tabla 2. Número de raíces comerciales (NRC), Número de Raíces No Comerciales (NRNC) y Número de Raíces Totales (NRT) por ha, Peso Fresco de Raíces Comerciales (PFRC), Peso Fresco de Raíces No Comerciales (PFRNC) y Peso Fresco de Raíces Totales (PFT) expresados en tn ha ${ }^{-1}$ de los cvs de batata cultivados en Corrientes, 2019/20.

\begin{tabular}{lcccccc}
\hline Cultivar & NRC & PFRC & NRNC & PFRNC & NRT & PFT \\
\hline BEA & $162500 \mathbf{a}$ & $31,76 \mathbf{a}$ & $167500 \mathbf{a}$ & $4,65 \mathbf{a}$ & $330000 \mathbf{a b}$ & $36,42 \mathbf{a b}$ \\
ARA & $170000 \mathbf{a}$ & $28,71 \mathbf{a}$ & $122500 \mathbf{a}$ & $4,32 \mathbf{a}$ & $292500 \mathbf{a}$ & $33,03 \mathbf{a}$ \\
MOR & $195000 \mathbf{a}$ & $48,21 \mathbf{a}$ & $170000 \mathbf{a}$ & $7,08 \mathbf{a}$ & $365000 \mathbf{a b}$ & $55,29 \mathbf{a b}$ \\
OKI & $277500 \mathbf{a}$ & $67,87 \mathbf{a}$ & $157500 \mathbf{a}$ & $4,91 \mathbf{a}$ & $435000 \mathbf{b}$ & $72,79 \mathbf{b}$ \\
\hline CV $(\%)$ & 29,62 & 43,00 & 19,99 & 26,58 & 18,45 & 38,20 \\
\hline
\end{tabular}

El NRC y el NRNC por ha no difirió estadísticamente entre los cultivares, pero la sumatoria de ambos, el NRT puso de manifiesto diferencias entre los cultivares que siguieron la misma respuesta que con las variables de los pesos de raíces (tn ha ${ }^{-1}$ ) antes mencionadas.

Ambos cultivares de ciclo corto, han presentado los menores valores de los componentes numéricos del rendimiento. Dentro de estos, el $c v$ Arapey INIA ha presentado los menores valores absolutos de las variables medidas. Los cultivares de ciclo largo, sobresalieron y particularmente el $c v$ Okinawa 100 alcanzó los mayores valores absolutos en términos asociados a la producción total.

La aplicación de tecnología simple y accesible como la fertilización adecuada a las demandas del cultivo, podría dar un plus de rendimiento que posibilita una mejora en los márgenes económicos. No obstante, se deben realizar investigaciones al respecto que aporten información acerca de las respuestas a diferentes fuentes de nutrientes y dosis.

La utilización de herbicida selectivo pre emergente mantuvo al cultivo libre de malezas durante los primeros 40 días posteriores a la plantación. Esta práctica podría resultar muy conveniente en planteos de escala don- 
de el desmalezado manual se torna muy costoso. Sin embargo, no mover el suelo durante este periodo, dificulta la aireación de los camellones y el crecimiento de las raíces. El herbicida post emergente selectivo puede ser un gran aliado a la hora de controlar gramíneas, sobre todo en estados avanzados del cultivo donde los entrelíneos ya están cubiertos y el control manual no es posible.

El material de propagación saneado y la utilización de un cultivar apto es una tecnología de bajo costo que ha permitido como mínimo duplicar y hasta casi quintuplicar el rendimiento promedio citado para la Provincia de Corrientes. El cv Okinawa 100 alcanza los máximos rendimientos del cultivo de batata en las condiciones agroecológicas del norte de Corrientes con $72,8 \mathrm{tn} \mathrm{ha}^{-1}$ de raíces totales y particularmente a 67,9 tn ha ${ }^{-1}$ de raíces comerciales.

En cuanto al riego, ha permitido mantener en condiciones óptimas de humedad al cultivo durante pequeños, pero no menos dañinos, periodos de sequía. También es probable que haya contribuido a alcanzar los altos valores de rendimientos sin embargo para tener certeza de ello es necesario realizar ensayos con un testigo a secano y con diferentes tipos de riego.

Finalmente, no caben dudas que el potencial de rendimiento de este cultivo es enorme, sobre todo si se lo analiza desde el contexto regional y social en el que se encuentra en manos de pequeños productores donde el aprovechamiento pudiera ser integral. Tanto para su comercialización como hortaliza como para su transformación en carne.

\section{BIBLIOGRAFÍA}

Ames, T., Smit, N.E.J.M., Braun, A.R., O'Sullivan, J.R. y Skoglun, L.G. (1997). Sweetpotato: major pests and nutritional disorders. International Potato Center, Lima, Perú.

Araujo, J.O., Carballo, M.S., Lezcano, R.P., Mambrin, A.G., Picolini, P.E., Pinto Ruíz, G.A. Roncaglia, L.M. y Solís, V.E. (2019). Recuperación y desarrollo productivo de los cultivos de batata y mandioca en la provincia de Corrientes. Consejo Federal de Inversiones. Ministerio de Producción de Corrientes. Informe Final. Disponible en: http://biblioteca.cfi.org.ar/documento/recuperacion-y-desarrolloproductivo-de-los-cultivos-de-batata-y-mandioca-en-la-provincia-de-corrientes/.

Arguedas Gamboa, P., Mora Molina, J., y Sanabria Mora, J. (2015). Comparación del contenido de carotenoides en productos nutracéuticos elaborados a partir de dos variedades de camote y yuca. Revista Tecnología en Marcha, 28 (4): 42-53. Disponible en: https:/www.scielo.sa.cr/pdf/tem/v28n4/03793982-tem-28-04-00042.pdf.

Delgado, M.A.S. y Serna, L.G.R. (2018). Aplicación de dos modalidades de riego por goteo en el crecimiento y producción de camote (Ipomoea batatas L., variedad INIA 320). Universidad Nacional Agraria La Molina. Lima. Perú. Anales Científicos Vol. 79 (1): 144-150.

Del Valle, M. D. (2017). Evaluación de fuentes de potasio sobre rendimiento de camote. Universidad Rafael Landívar. Facultad de Ciencias Ambientales y Agrícolas. Jacaltenango, Guatemala. Disponible en: http://recursosbiblio.url.edu.gt/tesisjcem/2017/06/14/Del\%20Valle-Marlo.pdf.

Dewis, J. y Freitas, F. (1970). Métodos físicos y químicos de análisis de suelos y aguas, Boletín No 10, FAO, Roma.

Di Feo, L.D.V. (2015). Producción, multiplicación y manejo de propágulos de batata de sanidad controlada. Córdoba, Argentina. Secretaría de Ciencia y Tecnología. Ministerio De Industria, Comercio y Desarrollo Científico Tecnológico. Gobierno de la Provincia De Córdoba.

Escobar, H. E., Ligier, H. D., Melgar, R., Matteio, H. y Vallejos, O. (1996). Mapa de suelos de la provincia de Corrientes. EEA INTA. Corrientes. Pp 434: 200-201.

FAOSTAT. (2019). Food and Agriculture Organization of the United Nations. Disponible en: http:// www.fao.org/faostat/en/\#data/QC/visualize.

Gauna, P.I. (2016). Guía para la producción de batata en Corrientes. 1a ed. Corrientes: Edicones INTA. Libro digital. https://inta.gob.ar/sites/default/files/inta___produccion_de_batata_en_corrientes.pdf 
Huamán, Z. (1992). Botánica sistemática y morfología de la planta de batata o camote. Boletín de Información Técnica 25, Centro Internacional de la Papa, Lima, Perú. 22pp. Disponible en: https://books.google.es/ books?

$\mathrm{id}=\mathrm{hIkspoOH} 9 \mathrm{NMC} \& \mathrm{printsec}=$ frontcover\&hl$=\mathrm{es} \&$ source $=\mathrm{gbs} \_\mathrm{ge} \_$summary_r\&cad $=0 \# \mathrm{v}=$ onepage $\& \mathrm{q}$ $\& \mathrm{f}=$ false .

León, B. Martínez, M. López, M. Rodríguez, L. Ardón, C. Rodríguez, I. Posas, F. y Vásquez, M. (2013). Manual de manejo del cultivo de camote. Programa PYMERURAL. Tegucigalpa, Honduras. Disponible en: https://es.calameo.com/read/002384628177f0b38b3a0.

Loebenstein, G., Thottappilly, G., Fuentes, S. y Cohen, J. (2009). Enfermedades por virus y fitoplasma. En El camote (pp. 105-134). Springer, Dordrecht.

Martí, H. (2008). La batata: oportunidades para una hortaliza subutilizada. Boletín Hortícola N 39: 14-17.

Martí, H., Parera, C. y Rodríguez, R. (2018). Producción de batata. Ediciones INTA. Buenos Aires. Disponible en:

https://www.researchgate.net/profile/Hector_Marti/publication/335501356_Produccion_de_Batata/ links/5d6929cf92851c85387eed2c/Produccion-de-Batata.pdf.

Marti, H.R., Mitidieri, M.S., Di Feo, L.D.V., Segade, G., Constantino, A., Chiandussi, M.C. y Filippi, M. (2014). Producción agroecológica de batata para el gran cultivo y la huerta familiar. . - $1^{\mathrm{a}}$ Ed. San Pedro, Buenos Aires: Ediciones INTA. Disponible en: https://inta.gob.ar/sites/default/files/script-tmpintasp-marti-et-al-manual-cultivo-de-batata-2014.pdf

Pletsch, R. (2006). El Cultivo de la Batata. Proyecto regional de pequeños y medianos productores. Ediciones INTA. EEA Corrientes. INTA. Agencia de Extensión Rural Corrientes.

SENASA. (1983). Servicio Nacional de Calidad y Sanidad Agroalimentaria. Resolución N 297. Ministerio de Agricultura ganadería y Pesca de la Nación. Bs. As. Argentina. Disponible en: http:// servicios.infoleg.gob.ar/infolegInternet/anexos/95000-99999/96677/texact.htm. 